\title{
Sensors with SOI FET Primary Transducer and Frequency Output
}

\author{
A.V. Leonov' ${ }^{1}$, A.A Malykh ${ }^{1}$, V.N. Mordkovich ${ }^{1}$, M.I. Pavlyuk ${ }^{2}$ \\ ${ }^{1}$ Institute of microelectronics technology and high purity materials RAS, b 6,Akademica Osip'yana \\ street, Chernogolovka, Moscow region, 142432, Russia \\ mord36@mail.ru \\ ${ }^{2}$ JSC "ICC Milandr", B 5, Georgievsky prospect, Moscow, Zelenograd, 124498, Russia
}

\begin{abstract}
:
It is shown, that dual-gate SOI FET with built-in channel and MISIM control system with two additional ohmic contacts to channel's lateral side that can be used as a sensing element of external actions microelectronic transducer with frequency output (magnetic field, temperature and ionizing radiation). Transducer can be formed by means of using a sensing element in autogenerator circuits of different types. Also self-oscillation of transistor channel current can be used, appearing at the area of electrons avalanche effect of volt-ampere characteristics. Electric mode changes of sensing element and MISIM system gates potential allows controlling frequency rate value of the transducer.
\end{abstract}

Key words: primary transducers, autogenerator, self-oscillator, double gate MISIM transistor, frequency output transformers

\section{Introduction}

Sustainable interest to external actions microelectronic transducer design with frequency output is determined by their obvious advantages in comparison to sensors, representing an output signal as voltage or current. Influence to frequency conversion allows improving sensor's response, upgrading their magnetic noise resistance, increasing operating frequency, simplifying analog to digital signal conversion. Besides, sensors with frequency output provide an opportunity of wireless (radio frequency) connection with indicator equipment blocks that is essential to the wide range of applications.

Influence to frequency conversion is often realized by autogenerator circuits, at the input the sensing element is switched, which electrical performance are changed under the external influence. It leads to generation frequency change proportionally to impact intensity. Conversion crucial condition is negative differential resistance of volt-ampere characteristic either of sensor or generator itself. Examples of microelectronic devices with frequency output conversing different impacts (ex. temperature, magnetic field, pressure) can be found in [1].

Since there is an obvious variation of used autogenerator circuits, it's necessary to notice common features of transducers, reviewed in [1].

1. To convert various external actions sensors of different construction and physical nature are used (piezo resistors to control pressure, thermo-resistor to control temperature, magnetoresistance and Hall's elements to control magnetic field).

2. To change autogenerator's basic frequency (i.e. frequency value without external action) one have to replace active or (and) passive elements, used in transducer's construction.

The main purpose of this work is to demonstrate opportunities of our SIO FET primary transducer [2] that could be used to convert different physical actions into frequency, and also allows varying transducer's basic frequency value and its dynamic range by means of electrical mode change.

\section{Sensing Element and Transducer Circuits}

The sensing element that we use is a dual-gate FET with embedded $n^{+}-n-n^{+}$channel and controlling Me - insulator - semiconductor insulator - Me (MISIM) structure. The transistor includes two additional ohmic $\mathrm{n}^{+}$contacts lying oppositely on the sides of the channel. A junctionless field MOS transistor and a resistor sensing element are integrated in such structure. The resistor sensing element responds to any external influences that change 
channel current (e.g. effects on magnetic field, temperature, pressure, radiation). Thus such sensing element is able not only to detect external effects but also to be involved in processing an induced signal.

Sensing element is fabricated by Si-on-Insulator technology. Transistor channel is formed in a thin $(0.1 \ldots 0.2 \mathrm{mkm}) \mathrm{Si}$ layer separated from the substrate by buried $\mathrm{SiO}_{2}$ dielectric inside it. At the same time, the Si substrate and buried dielectric layer are used as one of controlling MISIM system elements. The second field component is fabricated on the external channel area as in ordinary MOS transistor. The structure of the sensing element is shown on the fig. 1.

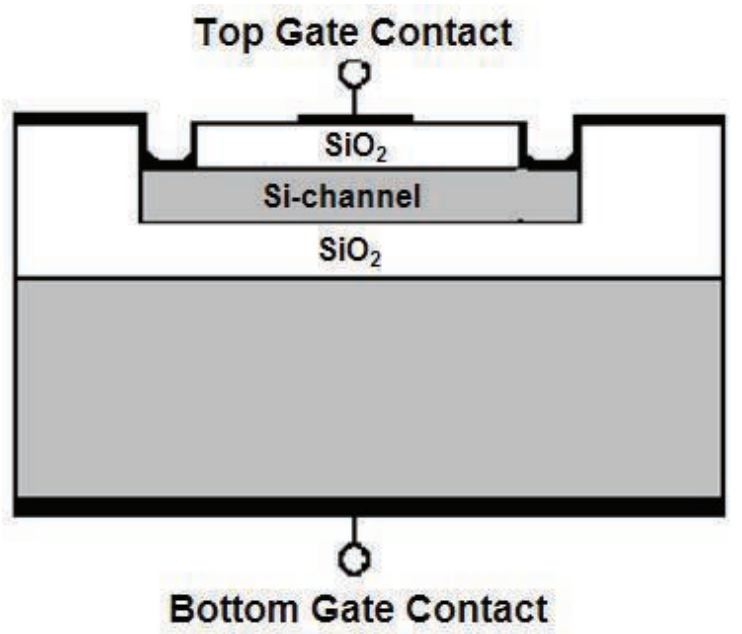

Fig. 1. SOI MISIM sensing element structure.

The article [2] points out that you can control many of performances of a sensing element (e.g. sensitivity dynamic range, temperature ratio figure, value of sensitivity unit, response to radiation) by changing its MISIM voltage supply and its MISIM gates potentials. The MISIM is particularly essential as it allows realizing negative or positive feedback between induced signal and controlling gates. We used these features of a sensing element in this work to convert induced signal into frequency.

Using a sensing element in influence frequency transducers we applied different autogenerator circuits. One of them is a circuit of negatron analog (fig. 2a), which is an ordinary flip-flop. The mode of the multivibrator is set by T5 and T6 transistors, which are controlled by the output of the sensing element proportional to intensity of external influence [1]. The circuit in the fig. $2 b$ is an autogenerator based on operational amplifier in which the sensing element is nonlinear. In this case, the base frequency is defined by capacitance $C$.

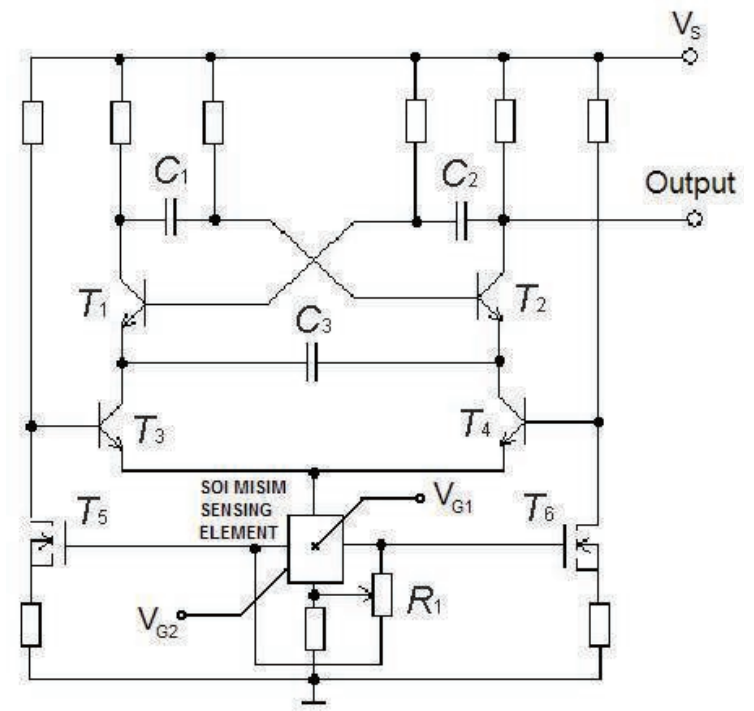

a.

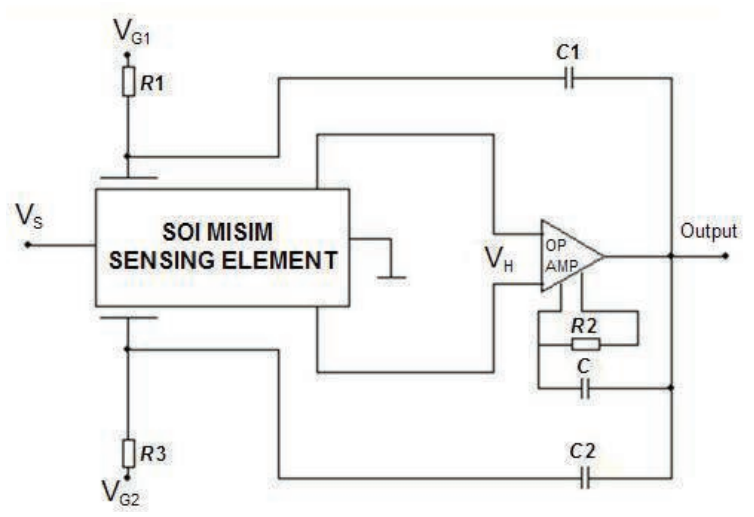

b.

Fig. 2. Autogenerator circuits using SOI MISIM sensing element.

\section{Base Conversion Frequency Controlling by Changing Electrical Mode of Sensing Element}

Electrical modes of a sensing element implies values of autogenerator voltage supply (voltage supply of a sensing element is included into the voltage supply autogenerator circuit) as well as potentials on controlling gates of the sensing element. At the same time, the base frequency can be changed both by voltage supply and gate potentials provided that values used in active and passive components' circuits are constant.

The fig. $3 a$ for the circuit of the autogenerator shown in the fig. $2 a$ points out that the base frequency of generation is linearly changed by 1.7 times by changing the supply voltage from 10 to $20 \mathrm{~V}$ while the positive gate potential values are constant. If the voltage supply is constant, behavior of autogenerator frequency depends on each gate potential. Fig. 3b illustrates the base frequency changing as a 
function of one gate, in case when supply voltage and another gate potential are constant. At the same time, simultaneous and identical potential changing on both gates of MISIM sensing elements leads to approximately twofold change of base frequency provided that gate potentials is less than voltage supply.

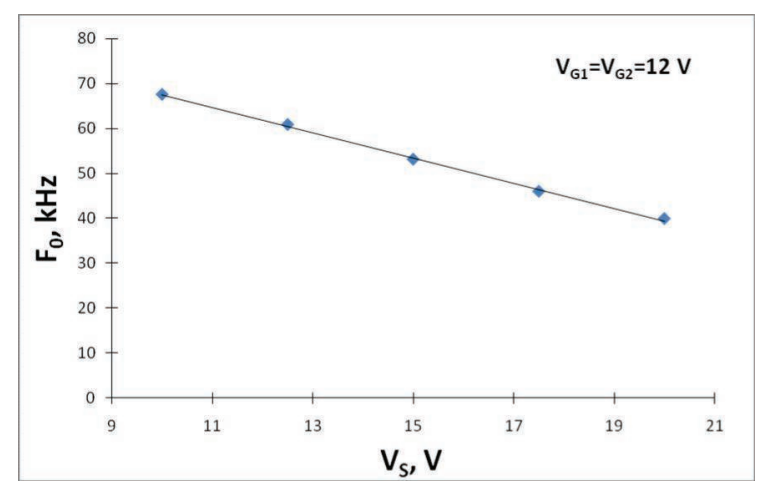

(a)

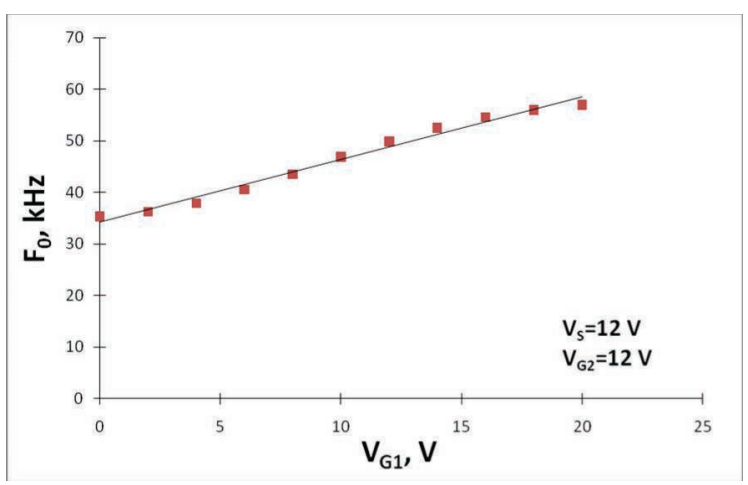

(b)

Fig. 3. Autogenerator base frequency controlling by changing electrical mode of SOI MISIM sensing element (a) by changing supply voltage (b) by changing gate potential.

\section{Magnetic Field Induction to Frequency Transducer}

The fig. 4 presents changing the autogenerator base frequency dependence on magnetic field induction (circuit fig. 2a). In this plots the base frequency without magnetic field is $50 \mathrm{kHz}$ while measurement interval of magnetic field induction is $5-50 \mathrm{mT}$.

One can see that when magnetic field intensity is growing, frequency changing is linearly increasing. The field density sensitivity in this case is approximately $100 \mathrm{~Hz} / \mathrm{mT}$ (fig. 4, curve 1). If we additionally include an IC amplifier in the circuit shown in the fig. $2 a$, between the hall contacts of the sensing element and T5 and T6 transistor gates, so to increase gate voltages of T5 and T6 proportionally to hall signal, we can increase magnetic field induction sensitivity of the transducer (fig. 4, curve 2).

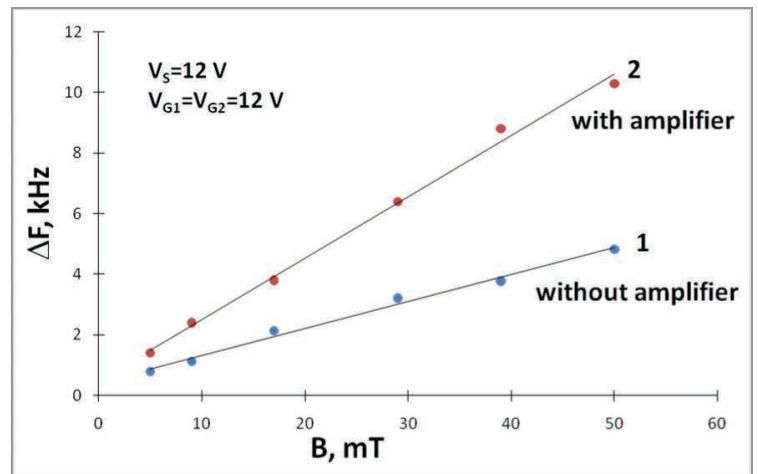

Fig. 4. Changing of the autogenerator base frequency dependence vs magnetic field induction intensity.

\section{Temperature to Frequency Transducer}

The transducer is built in accordance with the circuit shown in fig. $2 b$. To investigate the temperature effects on the MISIM base transducer frequency, sensing element was affected by thermal action at range of $20^{\circ} \mathrm{C}$ to $55^{\circ} \mathrm{C}$. The tab. 1 shows experimental values of the base frequency changes $(\Delta \mathrm{F})$ under the thermal action. The system sensitivity (S) was also estimated at different values of supply voltage and potentials, applied to MISIM gates of the sensing element.

Tab. 1: The dependence between the base frequency and transducer sensitivity on temperature at different values of supply voltage and potentials at MISIM gates of the sensing element

\begin{tabular}{|c|c|c|c|c|}
\hline $\mathrm{V}_{\mathrm{S}}, \mathrm{V}$ & $\mathrm{V}_{\mathrm{G} 1}=\mathrm{V}_{\mathrm{G} 2}, \mathrm{~V}$ & $\begin{array}{c}\Delta \mathrm{T}, \\
{ }^{\circ} \mathrm{C}\end{array}$ & $\begin{array}{c}\Delta \mathrm{F}, \\
\mathrm{Hz}\end{array}$ & $\begin{array}{c}\mathrm{S}, \\
\mathrm{Hz} /{ }^{\circ} \mathrm{C}\end{array}$ \\
\hline 8 & 10 & 25 & 150 & 6 \\
\hline 10 & 8 & 25 & 450 & 18 \\
\hline
\end{tabular}

The table shows, that in the second mode the transducer sensitivity value is three times higher than in the first. Thus, the supply voltage and potentials change, applied to MISIM gates, allows changing the sensitivity of temperaturefrequency transducer.

\section{Ionizing Radiation to Frequency Transducer}

The transducer is built in accordance with the circuit shown in fig. $2 b$. To investigate the influence of ionizing radiation on the output frequency characteristics, MISIM transistor was affected by $\mathrm{Y}$-quanta of ${ }^{60} \mathrm{Co}$ isotope. The radiation dose was $30 \mathrm{krad}$. During irradiation, the supply voltage and potential gates of MISIM system were absent. The tab. 2 shows comparative data of the sensor output frequency at different supply voltages and potentials at MISIM system gates, before and after $\mathrm{Y}$-quanta irradiation. It is clear, that the affection of ionizing radiation changes the 
transducer base frequency. It can also be seen, that with the increasing potentials, applied to MISIM transistor gates at $5 \mathrm{~V}$, the sensor Tab. 2: The affection of radiation on generation frequency and transducer sensitivity

\begin{tabular}{|c|c|c|c|c|c|}
\hline & $\mathrm{V}_{\mathrm{S}}, \mathrm{V}$ & $\mathrm{V}_{\mathrm{G} 1}, \mathrm{~V}$ & $\mathrm{~V}_{\mathrm{G} 2}, \mathrm{~V}$ & $\mathrm{~F}_{0}, \mathrm{kHz}$ & $\mathrm{S}, \mathrm{Hz} / \mathrm{rad}$ \\
\hline Basic & 10 & 15 & 15 & 28,13 & - \\
\hline Irradiated & 10 & 15 & 15 & 20,22 & 260 \\
\hline Basic & 10 & 20 & 20 & 21,39 & - \\
\hline Irradiated & 10 & 20 & 20 & 20,74 & 20 \\
\hline
\end{tabular}

\section{Temperature to Frequency Transducer Based on the MISIM Sensing Element Self Current Oscillations}

During the measurement of the volt-ampere characteristic of MISIM sensing element, it was discovered that at the initial section of the carriers avalanche multiplication in the channel (i.e. at the large longitudinal and transverse electric fields with the power supply of $\geq 28 \mathrm{~V}$ and gates potentials of $\geq 15 \mathrm{~V}$ ) there are current shown that these oscillations could also be used for conversion of external influence to frequency.

Structurally the transducer circuit with the effect described above, differs from the one shown in figure $2 \mathrm{~b}$ by the absence of autogenerator, because of exclusion of C-capacity from the circuit. The oscillating signal received at side contacts improves by operational amplifier and changes potential at the MISIM gates of the sensing element through the feedback circuit. Fig. 5 shows frequency change. It is clear, that the dependence is close to linear and the sensitivity is about $35 \mathrm{~Hz} /{ }^{\circ} \mathrm{C}$.

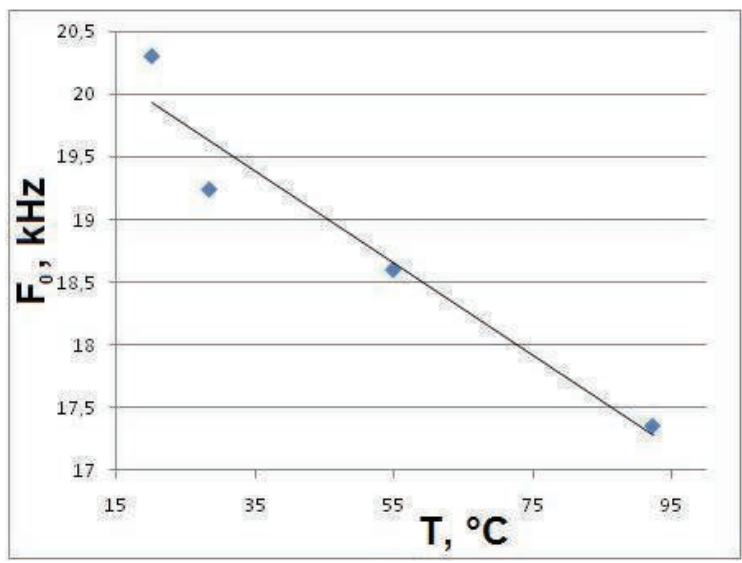

Fig. 5. Typical dependence of autogenerator base frequency vs temperature of temperature to frequency transducer using self current oscillations effect of SOI MISIM sensing element. oscillations between side contacts. It was sensitivity to radiation is considerably decreased.

\section{Conclusions}

It was shown above that the dual-gate field transistor SOI with the built-in channel and the controlling system MISIM with two additional ohmic contacts to lateral sides of the channel could be used as sensing element of microelectronic transducers of various external influences with frequency output. Transducers can be designed by using sensing element in autogenerator circuits of different types. Selfoscillations of the transistor's channel current can also be used. Preliminary studies have shown that besides the frequency conversion of magnetic field, temperature and Y-radiation, this sensing element can be also used in other different types of sensors with frequency output.

The objective of this work is not to get influence-frequency transducers with the highest characteristics. However, our experiments show that by optimizing electric modes of the sensing element, autogenerator constructions and properties of active and passive components used in circuits, it is possible to make transducers with high sensitivity (several units of $\mathrm{kHz} / \mathrm{mT}$ for magnetic field sensor, hundreds of $\mathrm{Hz} /{ }^{\circ} \mathrm{C}$ and $\mathrm{Hz} / \mathrm{rad}$ for temperature and radiation sensors), wide range of the base conversion frequency (from several $\mathrm{kHz}$ to several $\mathrm{MHz}$ ) and operation frequency stability not less than $1 \%$.

This work was done with partial support of the Russian Ministry of Education and Science (Agreement № 14.576.21.0026).

\section{References}

[1] O.A. Ageev, V.M. Manikonova, V.V. Petrov, V.N. Kotov, O.N. Negodenko, Microelectrinic transducers of non-electrical effects, Taganrog, TRTU publ., 2000, 153 p.(in Russian)

[2] M.L. Baranochnikov, A.V. Leonov, V.N. Mordkovich, D.M. Pazhin, The Features of Magnetosensitive Sensor Based on a Field Effect Hall Probe, Instruments and Experimental Techniques 55-6, 701-708 (2012); doi: $10.1134 / S 0020441212060024$ 